\title{
Effects of Temporary Functional Deafferentation on the Brain, Sensation, and Behavior of Stroke Patients
}

\author{
Elisabeth Sens, ${ }^{1}$ Ulrike Teschner, ${ }^{1,2}$ Winfried Meissner, ${ }^{3}$ Christoph Preul, ${ }^{2}$ Ralph Huonker, ${ }^{2}$ Otto W. Witte, ${ }^{2}$ \\ Wolfgang H. R. Miltner, ${ }^{1}$ and Thomas Weiss ${ }^{1}$ \\ ${ }^{1}$ Biological and Clinical Psychology, Friedrich Schiller University, Jena, D-07743, Germany, ${ }^{2}$ Department of Neurology, University Hospital, Jena, D-07747, \\ Germany, and ${ }^{3}$ Department of Anesthesiology and Intensive Care, University Hospital, Jena, D-07747, Germany
}

Following stroke, many patients suffer from chronic motor impairment and reduced somatosensation in the stroke-affected body parts. Recent experimental studies suggest that temporary functional deafferentation (TFD) of parts of the stroke-affected upper limb or of the less-affected contralateral limb might improve the sensorimotor capacity of the stroke-affected hand. The present study sought evidence of cortical reorganization and related sensory and motor improvements following pharmacologically induced TFD of the stroke-affected forearm.

Examination was performed during $2 \mathrm{~d}$ of Constraint-Induced Movement Therapy. Thirty-six human patients were deafferented on the stroke-affected forearm by an anesthetic cream (containing lidocaine and prilocaine) on one of the $2 \mathrm{~d}$, and a placebo cream was applied on the other. The order of TFD and placebo treatment was counterbalanced across patients. Somatosensory and motor performance were assessed using a Grating orienting task and a Shape-sorter-drum task, and with somatosensory-evoked magnetic fields. Evoked magnetic fields showed significant pre- to postevaluation magnitude increases in response to tactile stimulation of the thumb of the stroke-affected hand during TFD but not following placebo treatment. We also observed a rapid extension of the distance between cortical representations of the stroke-affected thumb and little finger following TFD but not following placebo treatment. Moreover, somatosensory and motor performance of the stroke-affected hand was significantly enhanced during TFD but not during placebo treatment. Thus, pharmacologically induced TFD of a stroke-affected forearm might improve the somatosensory and motor functions of the stroke-affected upper limb, accompanied by cortical plasticity.

\section{Introduction}

The global burden of stroke is immense (Feigin et al., 2009). Among consequences of stroke, motor impairment and reduced somatosensation are common. In recent years the beneficial effects of neurorehabilitative treatments and mechanisms of cortical plasticity have become more prominent in stroke rehabilitation (Hummel et al., 2005; Cramer et al., 2011; Grefkes and Fink, 2011). A new technique, able to modulate mechanisms of cortical reorganization by noninvasive means, is temporary functional deafferentation (TFD).

TFD has been demonstrated to improve somatosensory and motor functions of the homonym contralateral or neighboring ipsilateral body part and modulates their neural cortical representations. For example, TFD of the right hand by tourniquet-

\footnotetext{
Received Nov. 28, 2011; revised June 15, 2012; accepted June 23, 2012.

Author contributions: E.S., W.M., O.W.W., W.H.R.M., and T.W. designed research; E.S. and U.T. performed research; E.S., C.P., R.H., and T.W. analyzed data; E.S., U.T., W.M., C.P., R.H., O.W.W., W.H.R.M., and T.W. wrote the paper.

This research was supported by grants from the Interdisciplinary Centre of Clinical Research (IZKF) Jena to W.H.R.M. and T.W. Thanks to Stefan Clauss, Dr. Theresa Götz, and Tina Radtke for their contribution in MEG data acquisition and analyses, and to Dr. Jeremy Thorne for language advice and manuscript editing.

The authors declare no competing financial interests.

Correspondence should be addressed to Prof. Thomas Weiss, Biological and Clinical Psychology, Friedrich Schiller University, Am Steiger 3H1, Jena, D-07743, Germany. E-mail: thomas.weiss@uni-jena.de.

DOI:10.1523/JNEUROSCI.5912-11.2012

Copyright $\odot 2012$ the authors $\quad 0270-6474 / 12 / 3211773-07 \$ 15.00 / 0$
}

induced anesthesia resulted in a rapid improvement of the grip strength, tactile discrimination, and sensibility of the left hand and increased the neural activity in the right primary motor cortex (Björkman et al., 2004b). Rossini et al. (1994) additionally demonstrated an increase in neural dipole source activity and deepening of the cortical representation of the middle finger during ischemic anesthesia of the neighboring fingers, explained by unmasking of existing pathways in primary somatosensory cortex (SI). Furthermore, pharmacologically induced TFD of radial and median nerves resulted in significant improvements in sensory discrimination around the ipsilateral lip. These improvements were accompanied by a shift of the cortical representations of the ulnar skin area of the hand and lip into the deafferented representations of parts of the hand, and a significant decrease of intracortical inhibition within the muscle representation of the extensor digiti minimi muscle (Weiss et al., 2004). Similarly, Björkman et al. (2009) reported increased right hand sensitivity and an expansion of its representation within SI in healthy subjects during TFD of the right forearm by a local anesthetic cream.

A few experimental studies have focused on TFD in chronic stroke patients. For instance, Muellbacher et al. (2002) demonstrated improved hand motor function in stroke patients during pharmacologically induced anesthesia. Furthermore, tourniquetinduced anesthesia of the less-affected hand was shown to improve somatosensory sensibility (Voller et al., 2006) and the motor performance (Floel et al., 2004) of the affected hand. The improvement of 
motor function was attributed to reduced interhemispheric inhibition (Floel et al., 2008). Weiss et al. (2011) demonstrated beneficial effects of TFD on the somatosensory sensibility and motor capacity of the stroke-affected hand using pharmacologically induced TFD of the more-affected forearm during $1 \mathrm{~d}$ of Constraint-Induced Movement Therapy (CIMT; Miltner et al., 1999; Bauder et al., 2001; Langhorne et al., 2009). Together, these results suggest that TFD affects cortical reorganization and improves the somatosensory and motor ability of the stroke-affected upper limb. Previous reports of TFD in stroke patients however have mainly focused on interhemispheric cortical changes and plasticity of the primary motor cortex, and cortical reorganization in SI on the ipsilesional (contralateral) hemisphere has been less well studied.

In the present study we investigated changes of cortical activation in SI following pharmacologically induced TFD of the stroke-affected forearm during $1 \mathrm{~d}$ of CIMT, as well as its effects on stroke patients' somatosensory sensibility and motor capacity.

\section{Materials and Methods}

\section{Patients}

Thirty-six chronic stroke patients (18 females, 18 males) who attended CIMT at our treatment center at the Friedrich Schiller University participated in this study. Patients' characteristics (age, lesion site, etc.) are shown in Table 1. The procedure of the TFD experiment was extensively described to participants who then provided written informed consent. The procedure was conducted in accordance with the Helsinki Declaration on human experimentation and approved by the ethics committee of the Friedrich Schiller University.

\section{Experimental design}

Similar to a previous examination (Weiss et al., 2011), subjects participated in the present experiment on $2 \mathrm{~d}$ of the standard CIMT (Bauder et al., 2001). The experiment consisted of two separate treatments, the application of a cream that contained $20 \mathrm{~g}$ of a placebo agent, and a cream containing $20 \mathrm{~g}$ of a eutectic anesthetic emulsion composed of $2.5 \%$ lidocaine and $2.5 \%$ prilocaine (Emla, AstraZeneca). Both creams were applied to the volar side of the more-affected forearm covering an area of $50 \times 150 \mathrm{~mm}$ located $\sim 10 \mathrm{~mm}$ above the wrist and sheathed with an occlusive plaster. Each patient received both creams. The order of the two treatments was counterbalanced across patients according to the order of admission (even number-placebo first, odd number-TFD first). Creams were applied in the morning before the start of the baseline evaluation (t1) of magnetoencephalographic (MEG) measurements and sensory and motor capacity assessment, and remained on the arm until the end of the treatment evaluation on each day ( $\mathrm{t} 2)$. Subjects were told that they would receive two types of a local anesthetic cream designed to support the training. After the plaster was fixed to the forearm, baseline MEG recordings of somatosensory evoked magnetic fields (SEFs) and the tests outlined below were made (t1). The usual course of CIMT for $3.5 \mathrm{~h}$ then followed. After the CIMT session, the same tests and SEF recordings were performed again (t2; Fig. 1).

\section{Recording of SEFs}

SEFs were recorded using a whole-head 306 channel magnetoencephalograph (Elekta Neuromag). During SEF recordings, patients were lying on a bed inside a magnetically shielded room with their head placed in the mold of the dewar. Patients received $\sim 300$ non-noxious tactile stimuli on the phalanges of the thumb (D1) and 300 similar stimuli on the little finger (D5) of their stroke-affected hand using a pneumatically driven stimulator (Somatosensory Stimulus Generator, 4-D NeuroImaging Inc.). The stimulator consisted of a thin rubber membrane of $10 \mathrm{~mm}$ diameter enclosed in plastic housing that was fixed with adhesive plaster to the skin. The membrane pressed on the skin when expanded by an air pressure pulse ( $25 \mathrm{psi})$. Stimuli were applied in random order with an interstimulus interval ranging from 800 to $1600 \mathrm{~ms}(0.625-1.25 \mathrm{~Hz})$. The electro-oculogram (EOG) was recorded from two vertically positioned $\mathrm{Ag} / \mathrm{AgCl}$ electrodes to identify recording epochs with eye movement artifacts. Trials with eye movement artifacts and with EOG amplitudes exceeding $150 \mu \mathrm{V}$ were excluded from further analysis. SEFs were sampled at a rate of $1000 \mathrm{~Hz}$ and recorded with a $0.1-330 \mathrm{~Hz}$ bandpass filter. An epoch lasted $500 \mathrm{~ms}$, including a $100 \mathrm{~ms}$ prestimulus period. Off-line data analysis included Maxwell filtering (Maxfilter Version 2.0.21, using the time domain extension), a baseline correction from -90 to $0 \mathrm{~ms}$, a bandpass filter from 0.1 to $100 \mathrm{~Hz}$, and selection of $50 \%$ of all channels, i.e., channels over a single hemisphere. Before the SEF recordings, an individual Cartesian head coordinate system was defined with a threedimensional digitizer to locate each patient's head position in the MEG dewar and to match the MEG data with magnetic resonance images (MRIs). The $x$-axis connected the two periauricular points and the $y$-axis was represented by the perpendicular projection from the nasion to the midpoint of the $x$-axis. The $z$-axis was a line orthogonal to the crossing of the $x$ - and $y$-axis. Additionally, four position indicator coils were attached to the head and their locations digitized (Preissler et al., 2011).

An equivalent current dipole (ECD) model was used to estimate the location and strength of the sources using a spherical head model, based on the individual, T1 weighted MRI. The MRI recording was done on a 1.5 Tesla GYROSCAN system (Philips AG; 3D-Flash sequence, 256 sagittal MR images of $1 \mathrm{~mm}$ slice thickness, $256 \times 256$ matrix $(1 \times 1 \mathrm{~mm}$ in-plane resolution) covering the entire head). For one patient a MRI recording was not available, so the center of his sphere was set to the Cartesian coordinates $0,0,40(x, y, z)$.

The ECD of each stimulated finger was determined as the earliest maximal activity between 35 and $80 \mathrm{~ms}$ poststimulus, excluding a $35 \mathrm{~ms}$ delay of the pressure onset to the trigger. Dipole locations were accepted if the goodness-of-fit was better than $r=0.85$ and the dipole moment exceeded $5 \mathrm{nA} \mathrm{m}$. The Euclidean distance (ED) between the dipole locations of D1 and D5 was calculated for each patient and each MEG session to quantify cortical reorganization of both fingers following TFD. Changes in the amount of cortical activity were assessed by comparing the dipole moments of D1 for each MEG examination. The dipole moment and the ED were transformed to a percentage of the pretest value to standardize the data. MEG data were analyzed with DANA Software Release 3 (Elekta).

\section{Assessment of motor and sensory functions}

Von-Frey hair testing. Von-Frey hair testing (VFHT) was used to characterize the efficiency of TFD on mechanical thresholds of the moreaffected forearm. Thresholds for touch were tested at a point marked for VFHT assessment in the center of the occlusive bandage of the treated area of subjects' stroke-affected forearm. A von-Frey hair set (VF2 OptiHair 2; Marstock Nervtest) was used for sensory assessment. According to Rolke et al. (2006), a method of limits with five ascending and descending series of VFHT was used to determine tactile detection thresholds. VFHT was defined as the geometric mean of five suprathreshold and five infrathreshold values. A normal distribution of VFHT was achieved by logarithmic transformation of the parameter ( $\log _{2}$ units) as the forces of von-Frey hairs increase by a factor of 2 (Baumgärtner et al., 2002). 
Table 1. Characteristics of patients

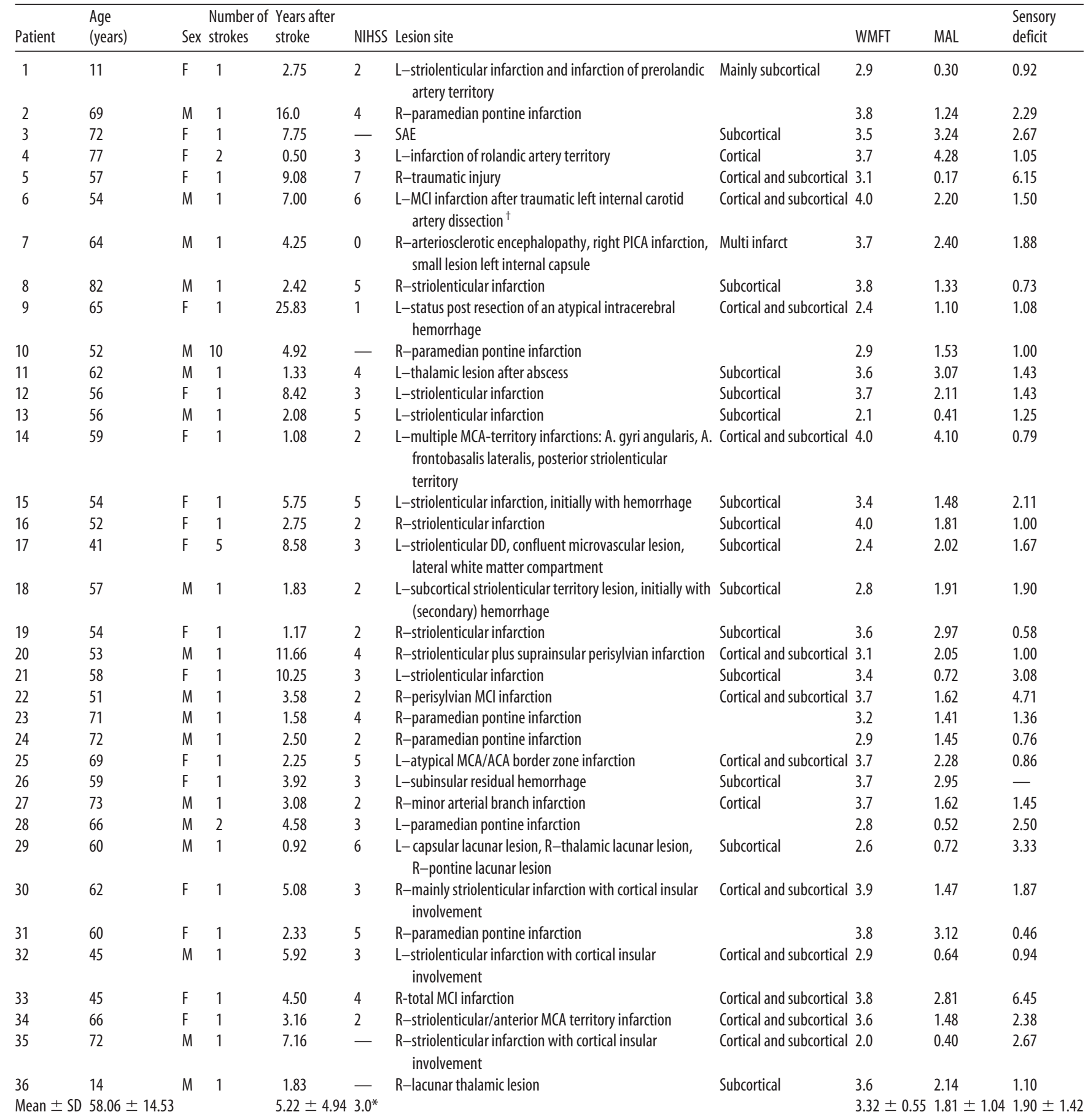

F, Female; M, male; L, left hemisphere; R, right hemisphere; NIHSS, NIH Stroke Scale; SAE, subcortical arteriosclerotic encephalopathy; MCl, middle cerebral artery; PICA, posterior inferior cerebellar artery; WMFT, Wolf Motor Function Test; MAL, Motor Activity Log; Sensory deficit, paretic hand/control hand.

*Median value.

${ }^{\dagger}$ No MRl, lesion site is based on previous diagnostic findings.

Grating orienting task. In accordance with a previous publication (Weiss et al., 2011), the grating orienting task (GOT) was used to measure limits of tactile resolution, using a modified technique adapted from Van Boven and Johnson (1994) (see also Craig et al., 2000; Tremblay et al., 2003; Bleyenheuft and Thonnard, 2007). We used a set of 14 hemispherical plastic domes with gratings cut into their surfaces, resulting in parallel bars and grooves of equal widths $(0.5-10 \mathrm{~mm})$ on each dome. Before measurement, patients were familiarized with the task by examining and testing the gratings. During sensory testing a cardboard screen was placed over the patients' arm to prevent viewing of their hand. Grat- ings were applied with the ridges and grooves randomly oriented in one of two orthogonal directions (perpendicular or parallel to the axis of the finger). Patients were asked to identify the alignment. We determined GOT thresholds, defined as the groove width at which responses were $75 \%$ correct.

Shape-sorter-drum task. The shape-sorter-drum task (SSDT) was used to measure movement performance (Weiss et al., 2011). Subjects were required to take 20 objects with their stroke-affected hand from a standard position and put them into a drum that contained several slots into which objects fitted according to their size and shape. Objects and slots 
Table 2. Mean and SD of raw data of the dependent variables VFHT, ED, dipole moment, SSDT, and GOT for the treatments TFD and placebo

\begin{tabular}{|c|c|c|c|c|c|}
\hline \multirow[b]{2}{*}{ Variable } & \multirow[b]{2}{*}{ Treatment } & \multicolumn{2}{|l|}{ Pre } & \multicolumn{2}{|l|}{ Post } \\
\hline & & Mean & SD & Mean & SD \\
\hline \multirow[t]{2}{*}{ VFHT } & TFD & 66.28 & 119.49 & 482.35 & 245.97 \\
\hline & Placebo & 59.39 & 81.49 & 74.61 & 112.81 \\
\hline \multirow[t]{2}{*}{ ED } & TFD & 10.90 & 4.86 & 11.95 & 4.26 \\
\hline & Placebo & 10.84 & 4.18 & 9.97 & 4.11 \\
\hline \multirow[t]{2}{*}{ Dipole moment } & TFD & 31.27 & 13.63 & 37.17 & 21.92 \\
\hline & Placebo & 33.51 & 19.81 & 30.67 & 13.39 \\
\hline \multirow[t]{2}{*}{ GOT } & TFD & 5.08 & 3.24 & 4.40 & 3.22 \\
\hline & Placebo & 4.87 & 3.19 & 4.80 & 3.08 \\
\hline \multirow[t]{2}{*}{ SSDT } & TFD & 335.80 & 341.64 & 320.26 & 346.93 \\
\hline & Placebo & 329.33 & 341.82 & 334.33 & 342.91 \\
\hline
\end{tabular}

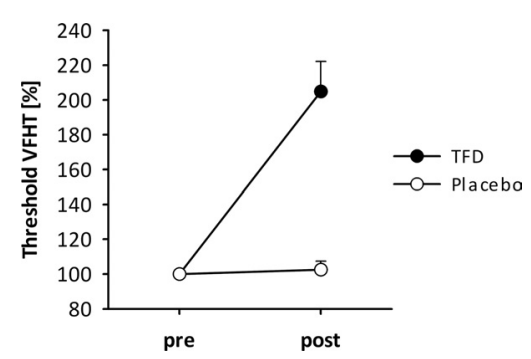

Figure 2. Changes and SEs in the VFHT thresholds at the forearm during TFD with anesthetic cream (black) and during placebo (gray). Performance is expressed as a percentage of pretest value; higher values indicate higher thresholds, i.e., less sensitivity.

were adapted to the abilities of subjects but were the same for a given subject on all measurements. The dependent variable, performance time, was measured from the start of movements to the instant the last object was successfully put into the drum, so shorter times would represent better performance. SSDT requires visual-motor as well as somatosensory-motor coordination to successfully pass the task.

\section{Statistical analysis}

After a logarithmic transformation of VFHT values, all mentioned outcome scales were interval-scaled. Therefore, repeated-measures ANOVAs with the within-subject factors Time ( $\mathrm{t} 1 \mathrm{vs} \mathrm{t} 2$ ) and Treatment (placebo vs anesthetic cream) were performed to assess differences in standardized ED, and dipole moment, and also to evaluate the outcome of SSDT, logarithmically transformed VFHT, and GOT as a function of TFD. Post hoc testing of significant results was performed using paired $t$ tests. We expected interactions between factors Time and Treatment. Linear relationship between the changes ( percentage of pretest values) in ED, dipole moment, GOT, SSDT, logarithmically transformed VFHT, and the sensory deficit of the index finger were assessed with Spearman correlation. Conditioned by missing normal distribution of some of these differences, nonparametric correlations were used. All statistical tests were performed with IBM SPSS Statistic for Windows (19.0) and the significance level was set to $p<0.05$.

\section{Results}

Mean values and SDs of raw data for the dependent variables VFHT, ED, Dipole Moment, GOT, and SSDT for the TFD and placebo treatments are presented in Table 2.

\section{Effectiveness of the TFD on VFHT}

We found significant main effects of factors Time $\left(F_{(1,35)}=98.19\right.$; $\left.p<0.001 ; \mathrm{\eta}^{2}=0.74\right)$ and Treatment $\left(F_{(1,35)}=40.25 ; p<0.001\right.$; $\left.\mathrm{n}^{2}=0.54\right)$ on VFHT scores, as well as a significant Time by Treatment interaction $\left(F_{(1,35)}=84.31 ; p<0.001 ; \mathrm{\eta}^{2}=0.71\right)$. The main effect of Time resulted from higher thresholds at $\mathrm{t} 2$ compared with $\mathrm{t} 1$ in both treatments. The main effect of Treatment indicates overall higher thresholds during TFD on the fore- arm following application of the anesthetic cream. Of interest is the Time by Treatment interaction that revealed no differences in VFHT between $\mathrm{t} 1$ and $\mathrm{t} 2$ for the placebo cream but significantly higher VFHT at $\mathrm{t} 2$ compared with $\mathrm{t} 1(t=-10.30$; $p<0.001$; Fig. 2 ) for the analgesic cream. Furthermore, there were no significant differences at $\mathrm{t} 1$ for the placebo compared with TFD.

\section{Neuromagnetic source imaging}

Twenty-nine of the 36 subjects fulfilled the criteria (goodness-offit $>0.85$, dipole moment $>5 \mathrm{nA} \mathrm{m}$ ) consistently over all conditions for the SEFs above the contralateral SI. Analysis of standardized values of ED for D1 and D5 dipoles revealed a significant factor Treatment $\left(F_{(1,28)}=4.82 ; p<0.05 ; \mathrm{y}^{2}=0.15\right)$ and a significant interaction of factors Time and Treatment $\left(F_{(1,28)}=\right.$ $\left.4.82 ; p<0.05 ; \mathrm{n}^{2}=0.15\right)$. Although the main effect of treatment resulted from larger ED during TFD, there were no significant differences of ED at $\mathrm{t} 1$ between the two treatments. However, post hoc analysis of the interaction revealed no significant change between $\mathrm{t} 1$ and $\mathrm{t} 2$ for the placebo treatment while ED increased significantly from $t 1$ to $t 2$ when subjects were treated with the analgesic cream $(t=-2,63 ; p<0.05$; Fig. $3 A)$.

In addition, we also analyzed changes in the amount of cortical activity based on standardized values of the dipole moment that represented activity of D1. ANOVA showed a significant factor Treatment $\left(F_{(1,29)}=7.98 ; p<0.05 ; \mathfrak{\eta}^{2}=0.22\right)$. The Treatment effect resulted from overall significantly stronger dipole moments during TFD than during placebo. There was a trend toward a main effect of factor Time $\left(F_{(1,29)}=3.14 ; p<0.09 ; \mathrm{y}^{2}=\right.$ $0.10)$ resulting from a trend toward higher values during $t 2$. Importantly, there was a significant interaction between Time and Treatment $\left(F_{(1,29)}=7.98 ; p<0.05 ; \mathrm{n}^{2}=0.22\right)$. Post hoc testing demonstrated that the dipole moment significantly increased from $t 1$ to 2 during TFD $(t=-2.46$; $p<0.05$; Fig. $3 B)$, whereas no change was observed when subjects were treated with placebo. Moreover, there was no significant difference at $\mathrm{t} 1$ between the two treatments.

\section{Sensory and motor functions}

Effectiveness of the TFD on GOT

ANOVA of the GOT data for the index finger revealed a main effect of factor Time $\left(F_{(1,35)}=4.79 ; p<0.05 ; \mathrm{y}^{2}=0.12\right)$ and a significant interaction between factors Time and Treatment $\left(F_{(1,35)}=11.229 ; p<0.01 ; \mathrm{y}^{2}=0.24\right)$. Regardless of treatment, better tactile resolution was observed at $\mathrm{t} 2$ compared with $\mathrm{t} 1$ (main effect of Time). The interaction indicates that the improvement of tactile resolution was stronger during TFD due to significantly better tactile resolution at $\mathrm{t} 2$ than at $\mathrm{t} 1$ when subjects were treated with the analgesic cream $(t=3.02 ; p<0.01$; Fig. 4$)$. However, no significant differences of tactile resolution were ob-
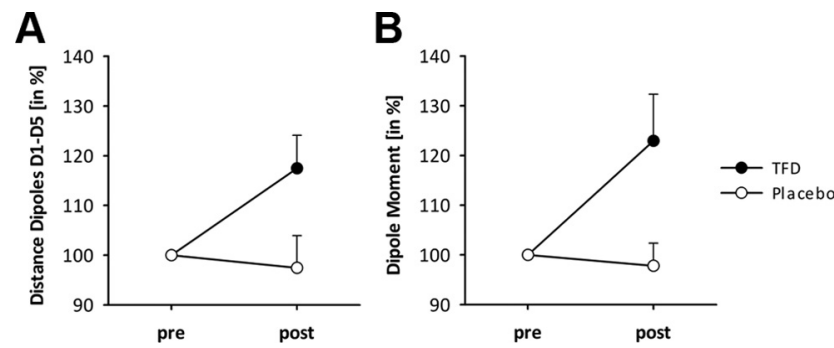

Figure 3. Changes and SEs in the ED between D1 and D5 $(\boldsymbol{A})$ and of the dipole moment for D1 (B) during TFD with anesthetic cream (black) and during placebo (gray). Changes are expressed as a percentage of pretest value. 


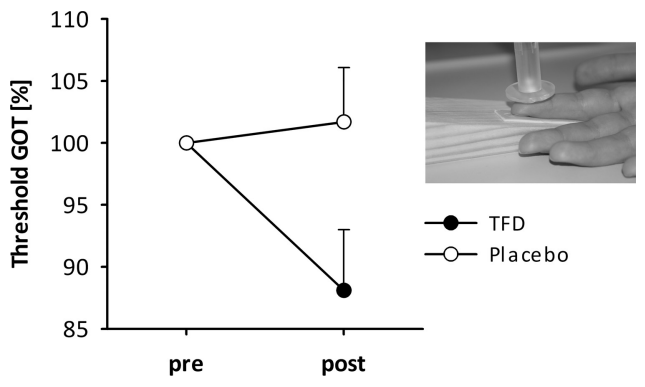

Figure 4. Thresholds and SEs in GOT performance during TFD with anesthetic cream (black) and with the placebo (gray) for the more-affected index finger. Performance is expressed as a percentage of pretest value; lower values indicate lower thresholds, i.e., better performance. The inset shows the task structure.

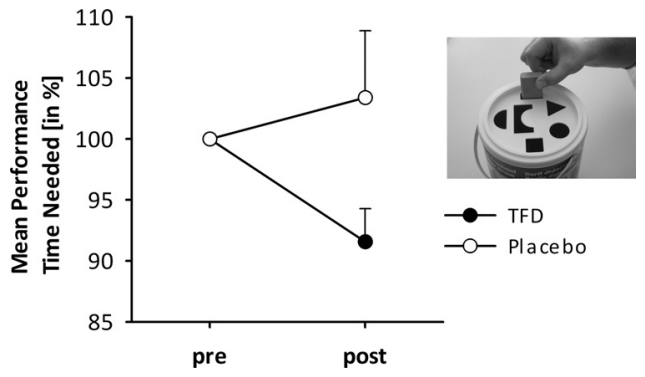

Figure 5. Mean performance (time needed and SES) for the SSDT during TFD with anesthetic cream (black) and during placebo (gray). Performance is expressed as a percentage of pretest value; lower values indicate lower thresholds, i.e., better performance. The inset shows the task structure.

tained at $\mathrm{t} l$ between the two treatments as well as between $\mathrm{t} 1$ and $\mathrm{t} 2$ when subjects were treated with the placebo. Furthermore, the index finger of the stroke-affected hand showed a significant sensory deficit (tactile resolutions, mean \pm SD: $4.83 \pm 3.09 \mathrm{~mm}$ ) compared with the index finger of the other hand $(2.82 \pm 1.26$ $\mathrm{mm} ; t=3.94 ; p<0.01)$. The results remain essentially the same when analyzing the 29 patients fulfilling the MEG criteria.

\section{Effectiveness of the TFD on SSDT}

ANOVA revealed no significant main effects of factors Time or Treatment (all $P$ values $>0.1$ ), but a significant interaction between factors Time and Treatment $\left(F_{(1,35)}=4.53 ; p<0.05 ; \mathrm{n}^{2}\right.$ $=0.12)$. Analysis of the interaction revealed no differences in performance times at $\mathrm{t} 1$ compared with $\mathrm{t} 2$ when subjects were treated with placebo cream, but significantly shorter performance times at $\mathrm{t} 2$ when subjects were treated with the analgesic cream compared with $\mathrm{t} 1(t=2.88$; $p<0.05$; Fig. 5). Moreover, there were no significant differences between placebo and TFD at t1. The results remain essentially the same when analyzing only the 29 patients fulfilling the MEG criteria.

\section{Relationship between parameters}

We examined the linear relationship between improvements in cortical activation, somatosensory capacity, and motor performance. All data were standardized with respect to the baseline value ( $\mathrm{t} 1)$. Changes in ED significantly correlated negatively with changes in SSDT $(r=-0.46 ; p<0.05$; Fig. 6$)$, i.e., a greater ED is related to better motor performance. There were no significant correlations between the increase of dipole moment, improvement of GOT, and improvement of SSDT.

We also tested whether the stroke-induced sensory deficits interact with the anesthetic effect of TFD. There was no cor-

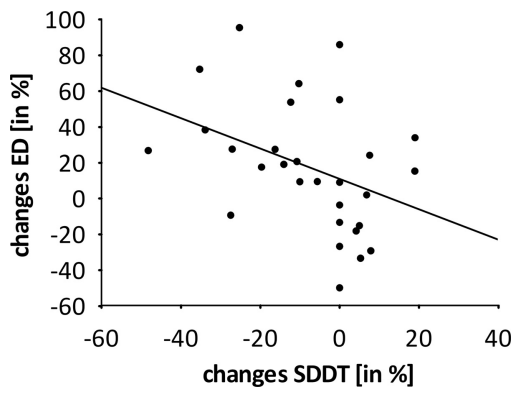

Figure 6. Scatter plot illustrating the relationship (negative correlation) between changes in SSDT scores and changes in ED during TFD. Changes are expressed as a percentage of pretest value.

relation between the anesthetic effect of the cream on the stroke-effected forearm and the sensory deficit of the index finger $(r=-0.13 ; p>0.01)$.

\section{Discussion}

The main findings of this study are threefold: (1) TFD of the stroke-affected forearm of chronic post-stroke patients led to cortical plasticity in the contralateral SI, i.e., the Euclidian distance between dipole locations of SEFs to stimulation of D1 and D5 increased from baseline to post-treatment following TFD but not following placebo treatment; (2) TFD led to an improvement of motor performance; and (3) TFD resulted in improved somatosensory sensitivity in the more-affected hand.

\section{Effectiveness of temporary functional deafferentation}

In the present study, we used a pharmacologically induced TFD on the stroke-affected forearm of chronic stroke patients to improve their sensory and motor abilities as well as to modulate cortical reorganization in the contralateral SI. TFD was highly efficient in reducing the somatosensory sensitivity of the affected forearm. Moreover, TFD with an anesthetic cream is an inexpensive technique with only minimal side-effects in the form of numbness in the affected forearm.

In contrast to other methods of deafferentation, TFD with an anesthetic cream has some advantages. Tourniquet-induced deafferentation is a painful procedure and leaves patients without motor control of the hand. Nerve blocks (e.g., brachial block) are invasive and often not tolerated by the patients, especially when applied on a daily basis in the clinical routine. In contrast, TFD with an anesthetic cream could be used during the whole training period of CIMT with longer follow-up periods. Rosén et al. (2006) showed improved sensory relearning in nerve-injured patients 6 weeks after a daily treatment with TFD over a period of 2 weeks. Hand motor practice during a single pharmacologically induced anesthesia resulted in improved motor function in stroke patients 2 weeks after treatment (Muellbacher et al., 2002). However, data on long-term effects of a prolonged application of TFD by an anesthetic cream are still missing for stroke patients.

\section{Effects of TFD on somatosensory-evoked magnetic fields}

TFD resulted in an increased ED of cortical sources of D1 and D5 for the stroke-affected hand in the contralateral SI. Larger distances were found within $5 \mathrm{~h}$ following TFD but not following placebo treatment. This observation might be due to the expansion of the hand area of SI into the representational area of the stroke-affected forearm whose sensory input was temporarily interrupted by TFD. This is in line with previous reports on healthy subjects where the neural representation area of the deafferented 
body part in SI was invaded by neighboring body parts (Weiss et al., 2004; Björkman et al., 2009). This result also corresponds to animal studies demonstrating short- and long-term changes in SI representation areas of different body parts following deafferentation (Merzenich et al., 1984; Calford and Tweedale, 1988, 1991; Pons et al., 1991).

TFD also resulted in a significantly increased dipole moment for the ECD of D1. This result is in line with previous findings in healthy subjects (Rossini et al., 1994; Björkman et al., 2004b). These changes might be explained by unmasking of existing silent connections based on a decrease of intracortical inhibition between adjacent cortical representations (Rossini et al., 1994; Liepert et al., 2004; Weiss et al., 2004; Floel et al., 2008), probably mediated through regulation of GABAergic transmission (Levy et al., 2002; Werhahn et al., 2002a). Thus, TFD might be used to influence and modulate cortical plasticity in stroke rehabilitation.

\section{Effects of TFD on sensory function}

TFD on the stroke-affected forearm significantly improved somatosensory sensitivity as measured by GOT. This is in accordance with previous results in healthy subjects demonstrating somatosensory improvements following TFD with anesthetic cream (Björkman et al., 2004a; Björkman et al., 2009) as well as with other kinds of TFD (Werhahn et al., 2002b; Weiss et al., 2004). Furthermore, different types of TFD were also shown to enhance the somatosensory sensibility of the stroke-affected hand in stroke patients (Voller et al., 2006; Weiss et al., 2011). As mentioned above, the observed improvements are probably based on rapid changes in the receptive fields at different levels of the somatosensory system, e.g., in the somatosensory cortex as well as in the thalamus (Nicolelis et al., 1993; Weiss et al., 2004; Jain et al., 2008; Björkman et al., 2009). Moreover, pharmacologically induced TFD on the right hand of healthy subjects was found to increase the central processing of the somatosensory system as indicated by increased somatosensory evoked potentials (Tinazzi et al., 2003). Overall, we suggest that TFD of the stroke-affected forearm is accompanied by rapid changes in the receptive fields of the somatosensory system. Our results suggest that TFD might enhance somatosensory capacities in chronic post-stroke patients.

\section{Effects of TFD on motor function}

In this study we also observed an improved motor performance as measured by SSDT during TFD compared with placebo treatment. Improving motor function is one of the main aims of neurorehabilitation for stroke patients (Langhorne et al., 2009; Cramer et al., 2011). A few experimental studies on TFD have addressed this problem and showed that increased somatosensory sensibility contributes to improvement of motor function in healthy subjects (Björkman et al., 2004b) as well as in post-stroke patients (Muellbacher et al., 2002; Floel et al., 2004; Floel et al., 2008). The results of this study are in line with our previous finding in stroke patients (Weiss et al., 2011) and demonstrate that TFD might help to improve motor functions.

\section{Relationship between parameters}

We found a significant relationship between cortical reorganization and motor function. Thus, an improved motor performance is associated with a larger distance of cortical sources of D1 and D5 in SI. Recent experimental studies demonstrated improved motor outcomes and changes in interhemispheric plasticity (Björkman et al., 2004b; Floel et al., 2004). In addition to these results we found a relationship between improved motor performance of the stroke-affected forearm and changes in the contralateral (ipsilesional) SI. This may be due to the high functional and anatomical interconnection between the motor and somatosensory system (Pavlides et al., 1993; van Meer et al., 2010).

We also expected an improvement in motor function associated with an increase of somatosensory sensibility. However, contrary to our hypothesis we did not find changes in GOT related to improvements in SSDT, thus no significant linear relationship between sensory and motor improvement. A reason for this unexpected result might lie in the variability of patients' lesions, including cortical, subcortical, and diffuse areas (Table 1). Further research is required to elucidate the impact of lesion site on this relationship. Alternatively, the motor and somatosensory system could independently benefit from TFD. While previous studies in healthy subjects (Björkman et al., 2004b) and nerveinjured patients (Björkman et al., 2005) have shown effects of TFD both on the motor and the somatosensory systems, the relationship between systems during TFD has not been investigated extensively in healthy subjects nor in chronic patients.

\section{Conclusion}

Our data show that a simple, inexpensive, pharmacological method of TFD of a stroke-affected forearm by an anesthetic cream results in cortical reorganization as well as in improvements of motor performance and somatosensory discrimination. After testing of its short-term efficiency, long-term effects of repeated TFD procedures across a period of several days are needed to ascertain whether this technique might become an additional tool in the improvement of motor rehabilitation in post-stroke patients.

\section{References}

Bauder H, Taub E, Miltner WHR (2001) Behandlung motorischer Störungen nach Schlaganfall. Die Taubsche Bewegungsinduktionstherapie. Göttingen: Hogrefe.

Baumgärtner U, Magerl W, Klein T, Hopf HC, Treede RD (2002) Neurogenic hyperalgesia versus painful hypoalgesia: two distinct mechanisms of neuropathic pain. Pain 96:141-151.

Björkman A, Rosén B, Lundborg G (2004a) Acute improvement of hand sensibility after selective ipsilateral cutaneous forearm anaesthesia. Eur J Neurosci 20:2733-2736.

Björkman A, Rosén B, van Westen D, Larsson EM, Lundborg G (2004b) Acute improvement of contralateral hand function after deafferentation. Neuroreport 15:1861-1865.

Björkman A, Rosén B, Lundborg G (2005) Enhanced function in nerveinjured hands after contralateral deafferentation. Neuroreport 16:517-519.

Björkman A, Weibull A, Rosén B, Svensson J, Lundborg G (2009) Rapid cortical reorganisation and improved sensitivity of the hand following cutaneous anaesthesia of the forearm. Eur J Neurosci 29:837-844.

Bleyenheuft Y, Thonnard JL (2007) Tactile spatial resolution measured manually: a validation study. Somatosens Mot Res 24:111-114.

Calford MB, Tweedale R (1988) Immediate and chronic changes in responses of somatosensory cortex in adult flying-fox after digit amputation. Nature 332:446-448.

Calford MB, Tweedale R (1991) Immediate expansion of receptive-fields of neurons in area- $3 \mathrm{~b}$ of macaque monkeys after digit denervation. Somatosens Mot Res 8:249-260.

Craig JC, Johnson KO, Johnston KO (2000) The two-point threshold: not a measure of tactile spatial resolution. Curr Direct Psychol Sci 9:29-32.

Cramer SC, Sur M, Dobkin BH, O'Brien C, Sanger TD, Trojanowski JQ, Rumsey JM, Hicks R, Cameron J, Chen D, Chen WG, Cohen LG, deCharms C, Duffy CJ, Eden GF, Fetz EE, Filart R, Freund M, Grant SJ, Haber S, et al. (2011) Harnessing neuroplasticity for clinical applications. Brain 134:1591-1609.

Feigin VL, Lawes CMM, Bennett DA, Barker-Collo SL, Parag V (2009) 
Worldwide stroke incidence and early case fatality reported in 56 population-based studies: a systematic review. Lancet Neurol 8:355-369.

Floel A, Nagorsen U, Werhahn KJ, Ravindran S, Birbaumer N, Knecht S, Cohen LG (2004) Influence of somatosensory input on motor function in patients with chronic stroke. Ann Neurol 56:206-212.

Floel A, Hummel F, Duque J, Knecht S, Cohen LG (2008) Influence of somatosensory input on interhemispheric interactions in patients with chronic stroke. Neurorehabil Neural Repair 22:477-485.

Grefkes C, Fink GR (2011) Reorganization of cerebral networks after stroke: new insights from neuroimaging with connectivity approaches. Brain 134:1264-1276

Hummel F, Celnik P, Giraux P, Floel A, Wu WH, Gerloff C, Cohen LG (2005) Effects of non-invasive cortical stimulation on skilled motor function in chronic stroke. Brain 128:490-499.

Jain N, Qi HX, Collins CE, Kaas JH (2008) Large-scale reorganization in the somatosensory cortex and thalamus after sensory loss in macaque monkeys. J Neurosci 28:11042-11060.

Langhorne P, Coupar F, Pollock A (2009) Motor recovery after stroke: a systematic review. Lancet Neurol 8:741-754.

Levy LM, Ziemann U, Chen R, Cohen LG (2002) Rapid modulation of GABA in sensorimotor cortex induced by acute deafferentation. Ann Neurol 52:755-761.

Liepert J, Weiss T, Meissner W, Steinrücke K, Weiller C (2004) Exerciseinduced changes of motor excitability with and without sensory block. Brain Res 1003:68-76.

Merzenich MM, Nelson RJ, Stryker MP, Cynader MS, Schoppmann A, Zook JM (1984) Somatosensory cortical map changes following digit amputation in adult monkeys. J Comp Neurol 224:591-605.

Miltner WHR, Bauder H, Sommer M, Dettmers C, Taub E (1999) Effects of constraint-induced movement therapy on patients with chronic motor deficits after stroke: a replication. Stroke 30:586-592.

Muellbacher W, Richards C, Ziemann U, Wittenberg G, Weltz D, Boroojerdi B, Cohen L, Hallett M (2002) Improving hand function in chronic stroke. Arch Neurol 59:1278-1282.

Nicolelis MAL, Lin RCS, Woodward DJ, Chapin JK (1993) Induction of immediate spatiotemporal changes in thalamic networks by peripheral block of ascending cutaneous information. Nature 361:533-536.

Pavlides C, Miyashita E, Asanuma H (1993) Projection from the sensory to the motor cortex is important in learning motor-skills in the monkey. J Neurophysiol 70:733-741.

Pons TP, Garraghty PE, Ommaya AK, Kaas JH, Taub E, Mishkin M (1991) Massive cortical reorganization after sensory deafferentation in adult macaques. Science 252:1857-1860.

Preissler S, Dietrich C, Meissner W, Huonker R, Hofmann GO, Miltner WH,
Weiss T (2011) Brachial plexus block in phantom limb pain: a case report. Pain Med 12:1649-1654.

Rolke R, Magerl W, Campbell KA, Schalber C, Caspari S, Birklein F, Treede RD (2006) Quantitative sensory testing: a comprehensive protocol for clinical trials. Eur J Pain 10:77-88.

Rosén B, Björkman A, Lundborg G (2006) Improved sensory relearning after nerve repair induced by selective temporary anaesthesia-a new concept in hand rehabilitation. J Hand Surg Br 31:126-132.

Rossini PM, Martino G, Narici L, Pasquarelli A, Peresson M, Pizzella V, Tecchio F, Torrioli G, Romani GL (1994) Short-term brain plasticity in humans-transient finger representation changes in sensory cortex somatotopy following ischemic anesthesia. Brain Res 642:169-177.

Tinazzi M, Rosso T, Zanette G, Fiaschi A, Aglioti SM (2003) Rapid modulation of cortical proprioceptive activity induced by transient cutaneous deafferentation: neurophysiological evidence of short-term plasticity across different somatosensory modalities in humans. Eur J Neurosci 18:3053-3060.

Tremblay F, Wong K, Sanderson R, Coté L (2003) Tactile spatial acuity in elderly persons: assessment with grating domes and relationship with manual dexterity. Somatosens Mot Res 20:127-132.

Van Boven RW, Johnson KO (1994) The limit of tactile spatial resolution in humans: grating orientation discrimination at the lip, tongue, and finger. Neurology 44:2361-2366.

van Meer MP, van der Marel K, Wang K, Otte WM, El Bouazati S, Roeling TA, Viergever MA, Berkelbach van der Sprenkel JW, Dijkhuizen RM (2010) Recovery of sensorimotor function after experimental stroke correlates with restoration of resting-state interhemispheric functional connectivity. J Neurosci 30:3964-3972.

Voller B, Flöel A, Werhahn KJ, Ravindran S, Wu CW, Cohen LG (2006) Contralateral hand anesthesia transiently improves post-stroke sensory deficits. Ann Neurol 59:385-388.

Weiss T, Miltner WHR, Liepert J, Meissner W, Taub E (2004) Rapid functional plasticity in the primary somatomotor cortex and perceptual changes after nerve block. Eur J Neurosci 20:3413-3423.

Weiss T, Sens E, Teschner U, Meissner W, Preul C, Witte OW, Miltner WHR (2011) Deafferentation of the affected arm a method to improve rehabilitation? Stroke 42:1363-1370.

Werhahn KJ, Mortensen J, Kaelin-Lang A, Boroojerdi B, Cohen LG (2002a) Cortical excitability changes induced by deafferentation of the contralateral hemisphere. Brain 125:1402-1413.

Werhahn KJ, Mortensen J, Van Boven RW, Zeuner KE, Cohen LG (2002b) Enhanced tactile spatial acuity and cortical processing during acute hand deafferentation. Nat Neurosci 5:936-938. 